\title{
APPLICATION OF THE HURD-MORI REACTION FOR THE SYNTHESIS OF CHIRAL 1,2,3- THIA(SELENO)DIAZOLE DERIVATIVES FROM (+)-3-CARENE AND $\alpha$-PINENE
}

\author{
Morzherin Yu.Yu., ${ }^{\text {a* }}$ Glukhareva T.V., "Mokrushin V.S.,", Tkachev A.V.," and Bakulev V.A." \\ a The Urals State Technical University, 620002 Ekaterinburg, Russian Federation. \\ Fax: +7 3432 745483, e-mail: morierine@htf.ustu.ru \\ ${ }^{b}$ Institute of Organic Chemistry, Sibirian Branch of the Russian Academy of Sciences, \\ 630090 Novosibirsk, Russian Federation, e-mail: atkachev@nioch.nsc.ru
}

\begin{abstract}
Chiral 1,2,3-thaidiazoles derivatives containing either cyclopropyl or cyclobutyl groups were synthesized from seco-derivatives of $(+)-3$-carene and $\alpha$-pinene by the Hurd-Mori reaction.
\end{abstract}

\section{Introduction}

$1,2,3-$ Thidiazoles are known to be useful synthons in organic synthesis $(4,5)$. Very active cotton defoliant and plant activator were found among them recently (1-3). In the course of our research work we were interested in functionalized chiral 1,2,3-thiadiazoles 1,2 as potential fungicides. To prepare 1,2,3-thiadiazoles 1,2 we used Hurd and Mori $(6,7)$ reaction. Oxo nitriles 3,4 prepared from natural monoterpenes $(+)-3$-carene 5 and $\alpha$-pinene 6 by know method (8) were taken of as starting reagents.

\section{Results and Discussion}

The semicarbazones 7, 8 were readily prepared from the corresponding oxo nitriles 3,4 by reaction with semicarbazide hydrochloride at room temperature. When compounds 7,8 were treated with thionyl chloride at room temperature in $\mathrm{CH}_{2} \mathrm{Cl}_{2}$ thiadiazoles $1 \mathrm{a}, 2 \mathrm{a}$ were formed in 78 and $60 \%$ yields. Only the products of the destruction of the ring were found if this reaction was carried out at higher temperatures.

Generally, both methylene and methyl groups of the substituted acetone moiety in compound 7 could take part in the cyclization process to form isomeric products 1a and $\mathbf{9}$. Nevertheless, we have shown that the reaction of oxo nitrile 7 with thionyl chloride proceeds with high regioselectivity on the methylene group to form thiadiazole 1a as the only product and isomeric 1,2,3-thiadiazole 9 is not detected. The formation of compound $\mathbf{1}$ is in agreement with a series of observations on reactivity of the substituted acetone moiety in compound 3: most of reactions involve just the methylene group rather the methyl - intramolecular cyclization (9), C-nitrosation (10), bromination (11). When treated with $\mathrm{SeO}_{2}$ in ethanol, semicarbazones 7 or $\mathbf{8}$ produce 1,2,3-selenodiazoles $\mathbf{1 b}$ or $\mathbf{2 b}$. The 1,2,3-selenodiazoles are very unstable and are decomposed at $30-40^{\circ} \mathrm{C}$ or at room temperature in various solvents. Mass-spectra of the $1,2,3-$ selenadiazoles contain picks of the molecular ions having the ratio of isotope line charactetic for the compound with one selenium atom. 
In contrast to optically active 3-carene derivative 3, for the preparation of seco-pinane derivatives we used racemic oxo nitrile 4 as ca. 3:1 mixture of cis/trans isomers. Formation of the products 8 and 2 with the same isomeric ratio indicates that no epimerization occurs during the reaction and compounds $\mathbf{8}$ and $\mathbf{2}$ can be prepared in optically active form provided that the starting $\alpha$-pinene is optically active.

Thus, we have demonstrated that a simple two-step syntheses of chiral 1,2,3-thiadiazoles 1a, 2a and 1,2,3-selenodiazoles $\mathbf{1 b}, \mathbf{2} \mathbf{b}$ are readily achieved using available oxo nitriles $\mathbf{3 , 4}$ as the starting materials. All the reactions proceed under very mild conditions to give the final products in high yields.

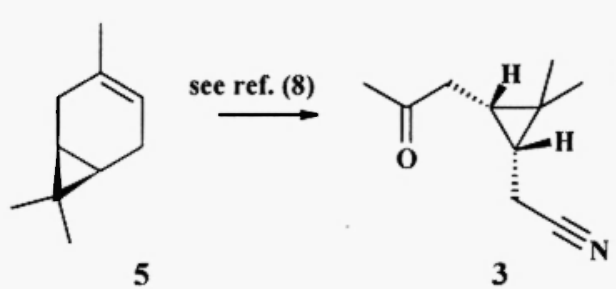<smiles>C/C=N/C(N)=O</smiles>

7

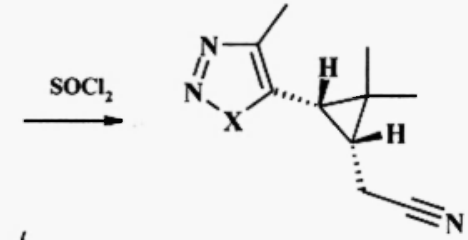

皮

$1 \mathbf{a , b}$

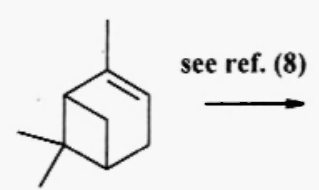

6<smiles>CC(=O)C1CC(CC#N)C1(C)C</smiles>

cis-trans $(3: 1)$

4

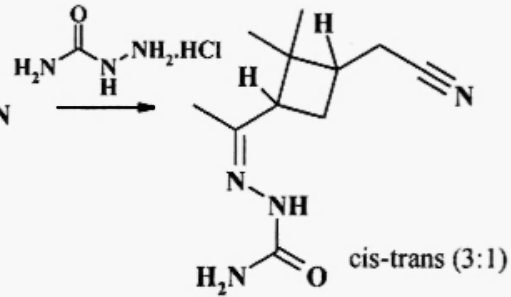

8

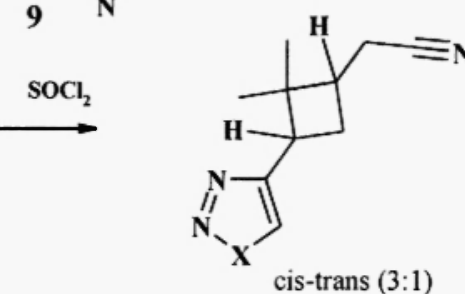

$\mathbf{2 a , b}$

$$
X=S(a), S e(b)
$$

\section{Experimental}

Unless other noted, chemicals were purchased from commercial suppliers and used without further purification. All solvents were distilled prior to use. Dry $\mathrm{CH}_{2} \mathrm{Cl}_{2}$ was prepared by distillation over $\mathrm{P}_{2} \mathrm{O}_{5}$ Analytical TLC plates were Silufol (Silpearl on aluminium foil). Preparative column chromatography was performed on $\mathrm{SiO}_{2}\left(0.140-0.315 \mathrm{~mm}\right.$, air dried and activated at $120^{\circ} \mathrm{C}$ for $\left.4 \mathrm{~h}\right){ }^{1} \mathrm{H}$ and ${ }^{13} \mathrm{C}$ NMR spectra were recorded in $\left[{ }^{2} \mathrm{H}_{6}\right] \mathrm{DMSO}$ and $\mathrm{CDCl}_{3}$ solutions on a Bruker DRX-500 $\left(500 \mathrm{MHz}\right.$ for ${ }^{1} \mathrm{H}$ and $125 \mathrm{~Hz}$ for $\left.{ }^{13} \mathrm{C}\right)$ and Bruker $\mathrm{WH}-250\left(250 \mathrm{MHz}\right.$ for $\left.{ }^{1} \mathrm{H}\right)$ using signals of the solvents as the internal standard $\left(\delta_{\mathrm{H}} 2.55 \mathrm{ppm}\right.$ and $\delta_{\mathrm{C}}$ $39.50 \mathrm{ppm}$ for $\mathrm{DMSO}, \delta_{\mathrm{H}} 7.24 \mathrm{ppm}$ and $\delta_{\mathrm{C}} 76.90 \mathrm{ppm}$ for $\mathrm{CDCl}_{3}$ ). IR spectra (in $\mathrm{KBr}$ ) were obtained using a UR-20 spectrometer. A Polamar A polarimeter was used to measure optical rotation at $578 \mathrm{~nm}$ at $30^{\circ} \mathrm{C}$. Mass spectra were obtained on a Finnigan MAT 8200 instrument using the electron impact ionization technique $\left(40-200^{\circ} \mathrm{C}, 70 \mathrm{eV}\right)$. New compounds $1 \mathrm{a}, 2 \mathrm{a}, 7,8$ gave satisfactory element analyses $\pm 0.35 \%$.

[(1R,3S)-2,2-Dimethyl-3-(2-oxopropyl)-cyclopropyl]-acetonitrile 3 and (3-acetyl-2,2-dimethylcyclobutyl)acetonitrile 4 (ca. 3:1 mixture of cis- and trans- isomers) were prepared by known method (8) via nitrosochlorination of (+)-3-carene and $\alpha$-pinene, respectively. 


\section{General procedure for the preparation of the simecarbazones}

A oxo nitriles 3 or 4 were added to an aqueous solution of 1 equivalent of semicarbazide hydrochloride. The reaction mixture was stirred at room temperature for 4 hours. The crystalline product was collected and dried under vacuum to give compounds $\mathbf{7}$ or $\mathbf{8}$ in 93 and $75 \%$ yield, respectively. The products were pure enough for further reactions. An analytical samples were prepared by crystallization from $95 \%$ aq. ethanol.

(1S,3R)-1-(2,2-Dimethyl-3-cyanomethylcycloprpyl)acetone semicarbazone 7: white powder, mp 182$184^{\circ} \mathrm{C} ; \mathrm{NMR}^{1} \mathrm{H}\left(\mathrm{DMSO}^{\mathrm{D}} \mathrm{D}_{6} \delta, \mathrm{ppm}, \mathrm{J} / \mathrm{Hz}\right): 8.86(1 \mathrm{H}, \mathrm{s}, \mathrm{NH}), 6.14\left(2 \mathrm{H}, \mathrm{s}, \mathrm{NH}_{2}\right), 2.5-2.6(2 \mathrm{H}, \mathrm{m}, \mathrm{CHCH}), 2.18$ $\left(2 \mathrm{H}, \mathrm{d}, 6.6, \mathrm{CH}_{2}\right), 1.80(3 \mathrm{H}, \mathrm{s}, \mathrm{Me}), 1.05(3 \mathrm{H}, \mathrm{s}, \mathrm{Me}), 0.96(3 \mathrm{H}, \mathrm{s}, \mathrm{Me}), 0.83\left(2 \mathrm{H}, \mathrm{d}, 6.9, \mathrm{CH}_{2}\right) . \mathrm{MS}, \mathrm{m} / \mathrm{z}(\%)$ : $223\left(\mathrm{M}^{+}, 1.7\right)$. IR (KBr), v/cm $\mathrm{cm}^{-1}: 3460,3270,3190(\mathrm{~N}-\mathrm{H}), 2960,2940,2860(\mathrm{C}-\mathrm{H}), 2230(\mathrm{C} \equiv \mathrm{N}), 1680(\mathrm{C}=\mathrm{O})$. Found, \%: $\mathrm{N} 24.84$. Calculated for $\mathrm{C}_{11} \mathrm{H}_{18} \mathrm{~N}_{4} \mathrm{O}, \%: \mathrm{N} 25.21$.

1-(2,2-Dimethyl-3-cyanomethylcyclobutyl)-methylketone semicarbazone 8 (ca. 3:1 mixture of cis- and trans- isomers): white powder, mp $200-202^{\circ} \mathrm{C}$; NMR ${ }^{1} \mathrm{H}\left(\mathrm{DMSO}-\mathrm{D}_{6}, \delta, \mathrm{ppm}, \mathrm{J} / \mathrm{Hz}\right): 8.86(1 \mathrm{H}, \mathrm{s}, \mathrm{NH}), 6.17$ $\left(2 \mathrm{H}, \mathrm{s}, \mathrm{NH}_{2}\right), 1.6-2.2\left(6 \mathrm{H}, \mathrm{m}, \mathrm{CHCH}_{2} \mathrm{CHCH}_{2}\right), 1.59(3 \mathrm{H}, \mathrm{s}, \mathrm{Me}), 1.22(3 \mathrm{H}, \mathrm{s}, \mathrm{Me}), 0.77(3 \mathrm{H}, \mathrm{s}, \mathrm{Me}) . \mathrm{MS}, \mathrm{m} / \mathrm{z}$ (\%): $223\left(\mathrm{M}^{+}, 2.7\right)$. IR (KBr), v/cm $\mathrm{cm}^{-1}: 3160(\mathrm{NH}), 2960(\mathrm{C}-\mathrm{H}), 2240(\mathrm{C} \equiv \mathrm{N}), 1680(\mathrm{C}=\mathrm{O})$. Found, \%: $\mathrm{N} 24,26$. Calculated for $\mathrm{C}_{11} \mathrm{H}_{18} \mathrm{~N}_{4} \mathrm{O}, \%$ : N 25,21.

\section{General procedure for the preparation of the thiadiazoles}

A suspension $0.5 \mathrm{~g}$ of 7 or 8 in dry dichloromethane $\left(20 \mathrm{~mL}\right.$ ) was treated with 20 equivalents of $\mathrm{SOCl}_{2}$ in one portion and stirred for $6 \mathrm{~h}$ at room temperature. Water $(20 \mathrm{~mL})$ was added, the organic phase was separated, dried with $\mathrm{Na}_{2} \mathrm{SO}_{4}$ and concentrated in vacuum. The oily residue was chromatographed on a silica gel column using hexane - ethyl acetate (1:2) as eluent to afford thiadiazoles 1a or $\mathbf{2 a}$.

4-Methyl-5-[(1S,3R)-2,2-dimethyl-3-cyanomethylcyclopropyl]-1,2,3-thiadiazole 1a: Yield $0.23 \mathrm{~g}$ (56\%); oil. $[\alpha]_{5 ; 8}^{30}+4.94$ (c $\left.0.0162, \mathrm{CHCl}_{3}\right)$. NMR ${ }^{1} \mathrm{H}\left(\mathrm{CCl}_{4}-\right.$ acetone- $\left.d_{6}, 4: 1 \mathrm{v} / \mathrm{v}, \delta \mathrm{H}, \mathrm{ppm}, \mathrm{J} / \mathrm{Hz}\right): 2.65(3 \mathrm{H}, \mathrm{s}$, $\left.M_{\text {thiadiazoie }}\right), 2.34(1 \mathrm{H}, \mathrm{dd}, 17.5,7.4, \mathrm{CHH}), 2.20(1 \mathrm{H}, \mathrm{dd}, 17.5$ and $8.0, \mathrm{CHH}), 2.00(1 \mathrm{H}, \mathrm{d}, 8.8, \mathrm{CH}), 1.58(1 \mathrm{H}$, ddd, 8.8, 8.0 and 7.4, $\left.\mathrm{CHCH}_{2}\right), 1.38(3 \mathrm{H}, \mathrm{s}, \mathrm{Me}), 1.15(3 \mathrm{H}, \mathrm{s}, \mathrm{Me}) . \mathrm{NMR}{ }^{13} \mathrm{C}\left(\mathrm{CDCl}_{3}, \delta, \mathrm{ppm}\right): 158.83\left(\mathrm{C}_{(5)}\right)$, $145.081\left(\mathrm{C}_{(4)}\right), 117.32(\mathrm{CN}), 27.94\left(\mathrm{Me}_{\text {thiadiazoie }}\right), 25.49(\mathrm{CH}), 22.95\left(\mathrm{CHCH}_{2}\right), 22.00\left(\mathrm{CMe}_{2}\right), 15.85(\mathrm{Me})$, $14.08\left(\mathrm{CH}_{2}\right), 12.67(\mathrm{Me})$. IR $\left(\mathrm{CCl}_{4}\right), v / \mathrm{cm}^{-1}: 2940,2900,2840(\mathrm{C}-\mathrm{H}), 2230(\mathrm{C}=\mathrm{N}) . \mathrm{UV}(\mathrm{EtOH}, \lambda \mathrm{nm}, \mathrm{lg} \varepsilon): 223$ (3.66), 266 (3.64). MS, $m / z(\%): 208.09029(\mathrm{M}+1,4.52)$. Calculated for $\mathrm{C}_{10} \mathrm{H}_{13} \mathrm{~N}_{3} \mathrm{~S}+\mathrm{H}$. 208.090. Found, \%: $\mathrm{N}$ 20.50; S 15.50. Calculated for $\mathrm{C}_{10} \mathrm{H}_{13} \mathrm{~N}_{3} \mathrm{~S}, \%: \mathrm{N} 20.27 ; \mathrm{S} 15.47$.

4-(2,2-Dimetyl-3-cyanomethylcyclobutyl)-1,2,3-thiadiazole 2a (ca. 3:1 mixture of cis- and trans- isomers): yield $0.28 \mathrm{~g}(60 \%)$; brown liquid. NMR ${ }^{1} \mathrm{H}\left(\mathrm{CDCl}_{3}, \delta, \mathrm{ppm}\right)$ : cis-isomer: $8.12\left(1 \mathrm{H}, \mathrm{s}, \mathrm{C}_{(5)} \mathrm{H}\right), 3.54(1 \mathrm{H}, \mathrm{dd}, \mathrm{J}=$ 10.4 and $7.6 \mathrm{~Hz}, \mathrm{CH}), 2.0-2.6\left(5 \mathrm{H}, \mathrm{m}, \mathrm{CH}_{2} \mathrm{CHCH}_{2}\right), 1.23(3 \mathrm{H}, \mathrm{s}, \mathrm{Me}), 0.62(3 \mathrm{H}, \mathrm{s}, \mathrm{Me})$; trans-isomer: 8.16 $\left(1 \mathrm{H}, \mathrm{s}, \mathrm{C}_{(5)} \mathrm{H}\right), 3.63(1 \mathrm{H}, \mathrm{dd}, \mathrm{J}=9.1$ and $6.2 \mathrm{~Hz}, \mathrm{CH}), 2.0-2.6\left(5 \mathrm{H}, \mathrm{m}, \mathrm{CH}_{2} \mathrm{CHCH}_{2}\right), 1.18(3 \mathrm{H}, \mathrm{s}, \mathrm{Me}), 0.65$ $(3 \mathrm{H}, \mathrm{s}, \mathrm{Me}) . \mathrm{NMR}{ }^{13} \mathrm{C}\left(\mathrm{CDCl}_{3}, \delta, \mathrm{ppm}\right)$ : cis-isomer: $162,4\left(\mathrm{C}_{(4)}\right), 131.5\left(\mathrm{C}_{(5)}\right), 118.3(\mathrm{CN}), 42.9\left(\mathrm{CMe}_{2}\right), 40.8$ $(\mathrm{CH}), 38.1\left(\mathrm{CHCH}_{2}\right), 29.1(\mathrm{Me}), 27.4\left(\mathrm{CH}_{2}\right), 17.1\left(\mathrm{CH}_{2} \mathrm{CN}\right), 17.0(\mathrm{Me})$; trans-isomer: $163.3\left(\mathrm{C}_{(4)}\right), 131.5\left(\mathrm{C}_{(5)}\right)$, $118.7(\mathrm{CN}), 41.1\left(\mathrm{CMe}_{2}\right), 40.3(\mathrm{CH}), 37.9\left(\mathrm{CHCH}_{2}\right), 27.0\left(\mathrm{CH}_{2}\right), 24.6(\mathrm{Me}), 23.2(\mathrm{Me}), 17.9\left(\mathrm{CH}_{2} \mathrm{CN}\right) . \mathrm{IR}$ $\left(\mathrm{CCl}_{4}\right), v / \mathrm{cm}^{-1}: 2950,2910,2850(\mathrm{C}-\mathrm{H}), 2230(\mathrm{C} \equiv \mathrm{N}) . \mathrm{UV}(\mathrm{EtOH}, \lambda \mathrm{nm}, \mathrm{lg} \varepsilon): 212(3.43), 261(3.25) . \mathrm{MS}, \mathrm{m} / \mathrm{z}$ (\%): $207.08544\left(\mathrm{M}^{+}, 6.49\right)$. Calculated for $\mathrm{C}_{10} \mathrm{H}_{13} \mathrm{~N}_{3} \mathrm{~S} 207.08301$. Found, \%: N 20.15; S 15.30. Calculated for $\mathrm{C}_{10} \mathrm{H}_{13} \mathrm{~N}_{3} \mathrm{~S}, \%$ : N 20,27; S 15,47. 


\section{General procedure for the preparation of the selenodiazoles.}

A suspension $0.5 \mathrm{~g}$ of 7 or 8 in ethanol $(20 \mathrm{~mL})$ was treated with 20 equivalents of $\mathrm{SeO}_{2}$ in one portion and stirred for $6 \mathrm{~h}$ at room temperature. After filtration the organic was concentrated in vacuum at room temperature. The oily residue was chromatographed on a silica gel column using hexane - ethyl acetate (1:2) as eluent to afford selenodiazoles $\mathbf{1 b}$ or $\mathbf{2} \mathbf{b}$.

4-Methyl-5-[(1S,3R)-2,2-dimethyl-3-cyanomethylcyclopropyl]-1,2,3-selenodiazole 1b: yield $0.38 \mathrm{~g}(67 \%)$; brown liquid. $\mathrm{NMR}^{1} \mathrm{H}\left(\mathrm{CDCl}_{3}, \delta, \mathrm{ppm}, \mathrm{J} / \mathrm{Hz}\right): 2.78\left(3 \mathrm{H}, \mathrm{d}, \mathrm{J}=0.9, \mathrm{Me}_{\text {selenodiazole }}\right), 2.44(1 \mathrm{H}, \mathrm{dd}, \mathrm{J}=17.1$ and 6.5 , $\mathrm{CHH}), 2.42(1 \mathrm{H}, \mathrm{dd}, \mathrm{J}=17.1$ and $7.9, \mathrm{CHH}), 2.20(1 \mathrm{H}, \mathrm{d}, \mathrm{J}=9.7, \mathrm{CH}), 1.67(1 \mathrm{H}, \mathrm{ddd}, \mathrm{J}=6.5,7.9$ and $9.7, \mathrm{CH})$, $1.39(3 \mathrm{H}, \mathrm{s}, \mathrm{Me}), 1.15$ (3H, s, Me). MS, $m / 2$ (\%): $214\left(\mathrm{M}-\mathrm{N}_{2}-\mathrm{Me}, 1.03\right), 212$ (5.29), 210 (2.63), 209 (1.02), 208 (1.09), 189 (1.85), 188 (1.11), 187 (10.19), 186 (1.90), 185 (6.47), 184 (3.04), 183 (2.92), 182 (1.04).

4-(2,2-Dimetyl-3-cyanomethylcyclobutyl)-1,2,3-selenodiazole 2b (ca. 3:1 mixture of cis- and transisomers): yield $0.38 \mathrm{~g}(67 \%)$; brown liquid. $\mathrm{NMR}^{1} \mathrm{H}\left(\mathrm{CDCl}_{3}, \delta, \mathrm{ppm}\right): 8.21 \cdot\left(1 \mathrm{H}, \mathrm{s}, \mathrm{CH}_{(5)}\right), 3.50-3.65(1 \mathrm{H}, \mathrm{m}$, $\mathrm{CH}), 2.0-2.6\left(5 \mathrm{H}, \mathrm{m}, \mathrm{CH}_{2} \mathrm{CHCH}_{2}\right), 1.28(3 \mathrm{H}, \mathrm{s}, \mathrm{Me}), 0.72(3 \mathrm{H}, \mathrm{s}, \mathrm{Me}) . \mathrm{MS}, \mathrm{m} / \mathrm{z}(\%): 227\left(\mathrm{M}-\mathrm{N}_{2}, 3.87\right), 225$ (2.02), 189 (12.61), 188 (1.35), 187 (6.93), 186 (2.88), 185 (4.53), 184 (1.25), 183 (1.59), 182 (1.32).

Acknowledgements: The authors thank the International Association for the Promotion of Cooperation with Scientists from the New Independent States of the Former Soviet Union (INTAS, Grant \# 97-0217) for the financial support of this work.

\section{References}

(1) N. Hanold, H. Kalbitz, M. Al-Smadi, H. Meier, Z. Naturforsch. 50b, 1121 (1995).

(2) K. Tsuji, H. Tsubouchi, K. Yasumura, M. Matsumoto, H. Ishikawa, Bioorg. Med. Chem. 4,2135 (1996).

(3) B. R. Babu; A. D. N. Vaz, Biochemistry 36, 7209 (1997).

(4) B. D'hooge; S. Smeets; S. Toppet; W. Dehaen, J. Chem. Soc., Chem. Commun. 1753 (1997).

(5) T. V. Glukhareva, Yu. Yu. Morzherin, V. S. Mokrushin, A. V. Tkachev, V. A. Bakulev, Khim. Geterotsikl. Soedin. 5, 707-708 (2000).

(6) C. D. Hurd; R. I. Mori, J. Amer. Chem. Soc. 77, 5359 (1955).

(7) P. Stanetty; M. Kremslehner, Heterocycles 48, 259 (1998).

(8) A.V. Tkachev; A.V. Rukavishnikov; A.M. Chibirjaev; L.B. Volodarsky, Synth. Commun. 20, 2123 (1990).

(9) A.V. Tkachev, A.V. Rukavishnikov, Mendeleev Commun., 2(4), 161 (1992).

(10) A.V. Rukavishnikov, A.V. Tkachev, Synth. Commun. 22(7), 1049 (1992).

(11) V.D. Kolesnik, A.V. Rukavishnikov, A.V. Tkachev, Mendeleev Commun., 5(5) 179 (1995).

\section{Received on February 17, 2001}

